\title{
Everybody was Kung-Fu fighting - The beneficial effects of Tai Chi Qigong and self-defense Kung-Fu training on psychological and endocrine health in middle aged and older men
}

\author{
Walther, Andreas ; Lacker, Tim J ; Ehlert, Ulrike
}

\begin{abstract}
Background: Higher age is associated to a variety of physical and mental disorders. Age-related changes in steroid secretion have been suggested to be an underlying mechanism leading to frailty, depression, and sexual dysfunction. However, Tai chi qigong and similar forms of exercise have been shown to improve a great variety of health-related parameters in older individuals. Methods: We examined 56 selfreporting healthy men actively practicing Tai chi qigong and/or self-defense Kung-fu and 55 age-matched self-reporting healthy controls. Saliva samples were obtained in a standardized procedure for subsequent quantification of circulating testosterone and cortisol levels. In addition, depressive symptoms, life satisfaction, and sexual health was assessesd via self-report questionnaires. Results: Age was negatively associated with testosterone, while no association emerged for cortisol. Tai chi qigong and/or self-defense Kung-fu training was neither associated with testosterone nor cortisol. More weekly Tai chi qigong and/or self-defense Kung-fu training (4 or more times per week) was instead associated with a lower CT-ratio, less depressive symptoms, and higher life satisfaction compared to individuals, who trained only one to three times per week. More years of Tai chi qigong and/or self-defense Kung-fu training were associated with less depressive symptoms and higher life satisfaction but not with the CT-ratio. No significant associations emerged for Tai chi qigong and/or self-defense Kung-fu training and sexual health. When compared to the age-matched controls, there is a significant effect of Tai chi, qigong and/or self-defense Kung-fu on the CT-ratio. Contrast analyses revealed a significantly lower CT-ratio for the high training load group in contrast to the low training load group. Further, in contrast to the control group, the low training load group exhibits a significantly higher CT-ratio. For depression, contrast analyses revealed a significantly lower level of depression in the high training load group compared to the control group. Conclusion: The results indicate that Tai chi qigong and/or self-defense Kung-fu training is beneficially associated with steroid secretion patterns and mental health in aging men, when training is performed with a frequency of 4 or more trainings per week. However, the high frequency training and control group show similar steroid secretion patterns suggesting an inverted U-shaped association between Tai chi qigong and/or self-defense Kung-fu training frequency and the CT-ratio in aging men. More research is needed to elucidate the underlying mechanism of this association. Still, Tai chi qigong and/or self-defense Kung-fu training provides a promising prevention strategy against age-related physical and mental deterioration in aging men.
\end{abstract}

DOI: https://doi.org/10.1016/j.ctim.2017.11.021

Posted at the Zurich Open Repository and Archive, University of Zurich

ZORA URL: https://doi.org/10.5167/uzh-143121

Journal Article

Accepted Version 
Originally published at:

Walther, Andreas; Lacker, Tim J; Ehlert, Ulrike (2018). Everybody was Kung-Fu fighting - The beneficial effects of Tai Chi Qigong and self-defense Kung-Fu training on psychological and endocrine health in middle aged and older men. Complementary Therapies in Medicine, 36:68-72.

DOI: https://doi.org/10.1016/j.ctim.2017.11.021 


\section{Accepted Manuscript}

Title: Everybody was Kung-Fu fighting. .. - The beneficial effects of Tai Chi Qigong and self-defense Kung-Fu training on psychological and endocrine health in middle aged and older men

Authors: A. Walther, T.J. Lacker, U. Ehlert

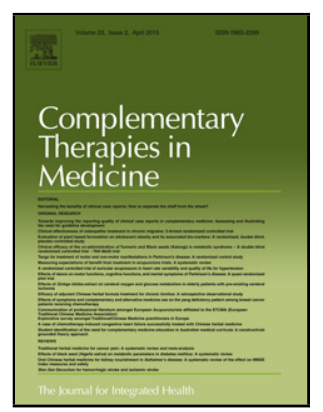

PII: S0965-2299(17)30231-5

DOI: https://doi.org/10.1016/j.ctim.2017.11.021

Reference: YCTIM 1778

To appear in: Complementary Therapies in Medicine

Received date: 5-4-2017

Revised date: 29-11-2017

Accepted date: 29-11-2017

Please cite this article as: Walther A, Lacker TJ, Ehlert U.Everybody was Kung-Fu fighting... - The beneficial effects of Tai Chi Qigong and self-defense Kung-Fu training on psychological and endocrine health in middle aged and older men.Complementary Therapies in Medicine https://doi.org/10.1016/j.ctim.2017.11.021

This is a PDF file of an unedited manuscript that has been accepted for publication. As a service to our customers we are providing this early version of the manuscript. The manuscript will undergo copyediting, typesetting, and review of the resulting proof before it is published in its final form. Please note that during the production process errors may be discovered which could affect the content, and all legal disclaimers that apply to the journal pertain. 


\title{
Article type:
}

Short report

\section{Running head:}

Tai Chi and steroid secretion in aging men

\section{Everybody was Kung-Fu fighting... - The beneficial effects of Tai Chi Qigong and self-defense Kung-Fu training on psychological and endocrine health in middle aged and older men}

\author{
Walther, A. ${ }^{1,2,}$, Lacker, T.J. ${ }^{1, \S} \&$ Ehlert, U. ${ }^{1 *}$ \\ ${ }^{1}$ Clinical Psychology and Psychotherapy, University of Zurich, Zurich, Switzerland \\ ${ }^{2}$ Biological Psychology, Technical University Dresden, Dresden, Germany \\ $\S$ These authors contributed equally to this publication
}

\section{Submitted to journal:}

Complementary Therapies in Medicine

\section{Correspondence:}

Prof. Dr. U. Ehlert

u.ehlert@psychologie.uzh.ch

\section{Hightlights:}

- Tai chi qigong is beneficially associated with steroid secretion in aging men.

- Tai chi qigong is beneficially associated with mental health in aging men.

- Tai chi qigong provides a promising prevention strategy for healthy aging in men.

\begin{abstract}
Background: Higher age is associated to a variety of physical and mental disorders. Agerelated changes in steroid secretion have been suggested to be an underlying mechanism leading to frailty, depression, and sexual dysfunction. However, Tai chi qigong and similar forms of exercise have been shown to improve a great variety of health-related parameters in older individuals.
\end{abstract}

Methods: We examined 56 self-reporting healthy men actively practicing Tai chi qigong and/or self-defense Kung-fu and 55 age-matched self-reporting healthy controls. Saliva samples were obtained in a standardized procedure for subsequent quantification of 
circulating testosterone and cortisol levels. In addition, depressive symptoms, life satisfaction, and sexual health was assessesd via self-report questionnaires.

Results: Age was negatively associated with testosterone, while no association emerged for cortisol. Tai chi qigong and/or self-defense Kung-fu training was neither associated with testosterone nor cortisol. More weekly Tai chi qigong and/or self-defense Kung-fu training (4 or more times per week) was instead associated with a lower CT-ratio, less depressive symptoms, and higher life satisfaction compared to individuals, who trained only one to three times per week. More years of Tai chi qigong and/or self-defense Kung-fu training were associated with less depressive symptoms and higher life satisfaction but not with the CTratio. No significant associations emerged for Tai chi qigong and/or self-defense Kung-fu training and sexual health. When compared to the age-matched controls, there is a significant effect of Tai chi, qigong and/or self-defense Kung-fu on the CT-ratio. Contrast analyses revealed a significantly lower CT-ratio for the high training load group in contrast to the low training load group. Further, in contrast to the control group, the low training load group exhibits a significantly higher CT-ratio. For depression, contrast analyses revealed a significantly lower level of depression in the high training load group compared to the control group.

Conclusion: The results indicate that Tai chi qigong and/or self-defense Kung-fu training is beneficially associated with steroid secretion patterns and mental health in aging men, when training is performed with a frequency of 4 or more trainings per week. However, the high frequency training and control group show similar steroid secretion patterns suggesting an inverted U-shaped association between Tai chi qigong and/or self-defense Kung-fu training frequency and the CT-ratio in aging men. More research is needed to elucidate the underlying mechanism of this association. Still, Tai chi qigong and/or self-defense Kung-fu training provides a promising prevention strategy against age-related physical and mental deterioration in aging men.

Key words: aging, tai chi, qigong, kung-fu, steroid secretion, testosterone, cortisol, depression, satisfaction with life, sexual function

\section{Introduction}

Aging is accompanied by an increased risk for physical and mental disease such as frailty, dementia, sexual dysfunction, or depression. ${ }^{1-3}$ As underlying mechanism contributing to this continuous health decline, age-related changes in steroid secretion have been suggested. ${ }^{4,5}$ In aging men testosterone (T), the end product of the hypothalamus-pituitary-gonadal (HPG) 
axis, continuously declines, ${ }^{6,7}$ while cortisol (C), the primary effector of the hypothalamuspituitary-adrenal (HPA) axis, continuously increases with age. ${ }^{8,9}$ Lower levels of $\mathrm{T}$ in men have been associated with depression, decreased life satisfaction, and sexual dysfunctions, ${ }^{10,11}$ while T-treatment showed significant improvements in mood and sexual function in older men. ${ }^{12}$ In contrast, higher levels of $\mathrm{C}$, and especially an elevated cortisol/testosterone (CTr) ratio reflecting the general (im)balance between the mutually inhibiting HPG and HPA axes, were shown to be associated with depression and overall worse health. ${ }^{13-15}$

Tai Chi is considered health protective and was originally used as combatting style but slowly transformed to an exercise form aiming to perform soft, flowing, and mindful movements, which require balance, strength, agility, coordination, concentration, and flexibility. Beneficial effects of Tai chi with regard to sleep disturbances or frailty have been shown for older adults. ${ }^{16,17}$ Tai chi can be combined with qigong, which is a self-healing system including meditation, breathing techniques, movements, and stands. Tai chi qigong can be practiced by people across all age groups and has recently been shown to be especially effective in improving self-reported psychosocial factors (e.g. loneliness) and functional health in elderly. ${ }^{18}$ In additon, 12 weeks of Tai chi qigong have been shown to reduce $\mathrm{C}$ levels in patients with chronic schizophrenia and female cancer survivors. ${ }^{19,20}$ Furthermore, a 12week Tai chi qigong program increased $\mathrm{T}$ levels in men aged 60 years or more with benign prostate hyperplasia. $^{21}$

However, to date the potential protective effects of longterm Tai chi qigong practice on endocrine, psychological, and sexual outcomes in healthy older men have not been investigated. Furthermore, classical selfe-defense systems (e.g. Wing-chun or Eskrima), which require similar skills as Tai chi qigong such as strength, balance, concentration, have never been investigated for their health protective potential in healthy aging men.

\section{Methods}

56 self-reporting healthy men between 40 and 75 years practicing Tai chi qigong and selfdefense Kung-Fu at the Suny Kamay Energy and Martial Arts Academy (SKEMA; $\underline{\text { www.skema.ch) }}$ and 55 age-matched self-reporting healthy male controls were recruited. This is a sub-sample of the Men's Health 40+ study fully described eslwhere. ${ }^{7}$ SKEMA is a Swiss martial arts academy providing standardized Tai chi qigong and self-defense trainings with a special focus on health maintenance in older individuals located in 25 different schools in Switzerland and Germany. The amount of years practicing Tai chi qigong and self-defense training (TYr) and the average amount of trainings per week (TWk) were used as continuous 
vairables. The movement mode of Tai chi qigong can be described as soft and flowing movement sequences combined with holding certain positions, which focus on breathing. Both, Tai chi and Qigong are regarded as therapeutic exercise forms, which can be combined to enhance the training effect. In contrast, self-defense Kung-fu and here especially SKEMA Kung-fu is a martial art form, which is trained in groups including exercises to strengthen the body but also performing blocking and attacking techniques in a slow and conscious manner and once mastered in a fast and automatic manner. Thereby, the whole body is used similarly to Tai chi qigong by focusing on the stands, leg-work, as well as the trunk, the arms, and the head. Finally, all is combined to flowing movement sequences, which are trained in pairs to simulate an opponents' attack and ones' defense.

Salivary $\mathrm{C}$ and $\mathrm{T}$ were obtained via standardized saliva sampling between 8:00-8:15am and subsequent biochemical analyisis at the biochemical laboratory of the Department of Psychology of the University of Zurich. As prior suggested, C and T were log transformed to subsequently calculate the CT-ratio. ${ }^{14}$ To measure depressive symptoms, the German version of the Center for Epidemiological Studies-Depression scale (CES-D) was used. ${ }^{22,23}$ Satisfaction with life was measured with the Satisfaction With Life Scale (SWLS), ${ }^{24}$ while sexual function was measured with the International Index of Erectile Function. ${ }^{25,26}$ Pearson bivariate correlations and partial correlations were used for data analysis. The level of significance was set at $\alpha=.05$. A set of potential covariates was used in the partial correlation analyses including age, fat and muscle mass in percent, medication or other drug intake, smoking status, coffee consumption, general health perception, having had a cold within the last two weeks, and having had a gumbleeding during the last days. Ethical approval for the study protocol was given by the local ethics committee of the University of Zurich. All subjects provided a written consent for their participation in the study.

\section{Results}

Sample characteristics are presented in table 1. The 35 individuals in the group with one to three trainings per week have an average age of $51.83(\mathrm{SD}=8.83)$ years, while those in the group with four or more trainings per week show a mean age of $45.9(\mathrm{SD}=8.83)$ years. The BMI for the group with less trainings per week is $25.1(\mathrm{SD}=2.33)$ and for those with more training sessions per week BMI was 25.9 ( $\mathrm{SD}=3.81$ ). 88.6\% of the group with fewer trainings per week reported to be non-smokers, while $66.7 \%$ in the group with more trainings per week reported to be non-smokers. For the healthy control group, average age was 49.95 
( $\mathrm{SD}=7.469)$, BMI was $25.93(\mathrm{SD}=3.77), \mathrm{C}$ was at average at $15.51 \mathrm{nmol} / \mathrm{l}(5.92), \mathrm{T}$ was at average at $77.84 \mathrm{nmol} / \mathrm{l}(\mathrm{SD}=27.83)$. The log-transformed $\mathrm{CTr}$ was at $-1.63(\mathrm{SD}=0.48)$. Ten participants took medication, whereas 45 did not. There were 42 non-smokers and 13 smokers in the control group. Eight of the participants in the control group did less than an hour of exercise per week and 47 engaged in 1-3 hours of physical exercise per week. For the Asian martial arts group, age was negatively associated with $\mathrm{T}(r=-.418, p=.001)$, while for $\mathrm{C}$ no association emerged $(r=-.016, p=.456)$. However, higher age was associated with a higher CT-ratio $(r=.253, p=.035)$. Partial correlation analysis revealed a significant association for TWk and CT-ratio $(r=-.261, p=.045)$, but not for TYr $(r=-.152, p=.166)$. However, with regard to T correlation coefficients were positive for TWk $(r=.122, p=.217)$ and TYr $(r=$ $.047, p=.382$ ), but did not reach statistical significance. Correlation coefficients for the association between C and TWk $(r=-.096, p=.271)$ and TYr $(r=-.117, p=.227)$ were negative, but did not reach statistical significance either. Depressive symptoms were negatively associated with $\operatorname{TYr}(r=-.337, p=.010)$ and a negative trend emerged for TWk $(r$ $=-.212, p=.077)$. Life satisfaction was positively associated with $\operatorname{TYr}(r=.360, p=.006)$ and TWk $(r=.350, p=.008)$, while for sexual dysfunction a negative trend emerged for TWk $(r=-.207, p=.087)$, but not for TYr $(r=-.059, p=.351)$. Further, t-tests for independent samples for CT-ratio and depressive symptoms were performed to investigate the differences between participants having 1-3 trainings per week and 4 or more trainings per week (Fig. 1). Results showed a significant difference $(t(51)=2.24, p=.019)$ for the CT-ratio but not for depressive symptoms $(t(53)=1.96, p=.055)$. The significant result points towards a lower CTratio for participants having a higher training load per week. Correlation analyses for the merged sample of healthy controls including the Asian martial arts groups showed a significant negative correlation with $\mathrm{T}(r=-.370, p<.001)$, no correlation with $\mathrm{C}(r=-.024, p=$ $.815)$ and a significant positive correlation with $\mathrm{C} \operatorname{Tr}(r=.262, p=.009)$, taken together the results support the results of the sole Asian martial arts group.

Additionally to the t-tests for independent samples for the two training groups, a group comparison with the healthy age-matched control group with almost equal sample size $(\mathrm{N}=$ 55), was conducted. The control group engaging in either less than one hour or one to three hours of physical activity per week and was not practising Tai chi, Qigong or Kung-Fu. The oneway ANOVA showed a significant effect of Tai chi, Qigong and self-defense Kung-Fu training on the CT-ratio $(F(2,95)=4.992, p=.009)$. A quadratic trend could be detected $(F(1,95)=9.284, p=.003)$, showing low CT-ratio for the control group and the high training load martial arts group, but a higher CT-ratio for the low training load martial arts group. 
Results for $\mathrm{C}(F(2,95)=1.518, p=.224), \mathrm{T}(F(2,95)=828.036, p=.369)$, depression scores $(F(2,107)=2.194, p=.116)$ and satisfaction with life $(F(2,107)=1.563, p=.214)$ were not significant. Further, planned contrasts were calculated to test differences between the control and martial arts group in general, between the control group and the high training load martial arts group, and the control group and low training load group. Results for the comparison between control and the martial arts groups showed a trend towards significance for depression scores $(t(107)=-1.817, p=.072)$ and satisfaction with life $(t(107)=1.657, p=.100)$ but no significant effects for CT-ratio $(t(95)=1.324, p=.189), \mathrm{C}(t(95)=1.078, p=.284), \mathrm{T}$ $(t(100)=-.503, p=.616)$. Planned contrasts between the control group and the high training load martial arts group showed significantly lower scores of depression $(t(107)=2.095$, $p=.039)$ and a trend towards higher satisfaction with life $(t(107)=-1.749, p=.083)$ but no significant differences for CT-ratio $(t(95)=0.252, p=.801), \mathrm{C}(t(95)=-.268, p=.789)$, or $\mathrm{T}$ $(t(100)=-.200, p=.822)$. The comparison between the control group and the low training load martial arts group showed a significant higher CT-ratio for the low training load martial arts group $(t(95)=2.888, p=.005)$ and a trend in the same direction for $C(t(95)=1.714, p=.090)$, but no significant results for $\mathrm{T}(t(100)=-1.260, p=.210)$, depression scores $(t(107)=-.572, p=.568)$ or satisfaction with life $(t(107)=0.756, p=.444)$. In addition, there was no significant difference in BMI between the groups $(F(2,100)=2.119, p=.126)$. Nonparametric tests comparing the control group and the Tai chi, Qigong and self-defense Kung-Fu group showed no significant difference in smoking status either $(\mathrm{U}=1441.5, p=.419)$.

\section{Conclusion}

More trainings per week in Tai chi qigong and self-defense Kung-fu were associated with a lower CT-ratio, less depressive symptoms, and a higher life satisfaction, while for more years of training less depressive symptoms and a higher life satisfaction was observed. In comparison with a healthy control group, not practicing Tai chi, Qigong or Kung-Fu, participants with a low Asian martial arts training load exhibited the highest CTr, whereas the control group exhibits comparably low CTr than the high training load group.

The results of the comparison between high and low traning load in Asian martial arts point to the conclusion that training in Tai chi qigong and self-defense Kung-fu might influence both, HPA and HPG activity in aging men by decelerating C-increase and Tdecline. However, training (TYr and TWk) was not significantly associated with T nor C, but with the CT-ratio an integrated endocrine parameter including variance of both hormones.

However, taking a healthy control group into account, the conclusions become more 
puzzling. The results, could be explained through a beneficial $\mathrm{C}$ increase in reaction to exercise, which increases the $\mathrm{CTr}$ without negative consequences, called the exerciseglucocorticoid paradox ${ }^{27}$. The decreased $\mathrm{C} T r$ for higher training load can be explained through a simultaneous incerase in $\mathrm{T}$, which, in turn, decreases the $\mathrm{CTr}^{28}$. Further, supporting this interpretation, depression scores in the low training load martial arts group are lower than in the control group, although these differences are not significant. Satisfaction with life is highest in the high training load group, with a trend towards statistical significance. Another explanation for the low $\mathrm{CTr}$ in the control group could be an increase in sex steroids as reaction of low intensity physical activity in low active individuals, followed by this increase, the CTr would decrease to a similar range than in the high training load martial arts group, but with a different underlying mechanism ${ }^{28}$. Replicating these analyses with a larger sample size might lead to significant differences between the control and Asian martial arts groups, since the direction is as expected and a trend towards statistical significance can be detected.

Keeping in mind the small sample sizes, the effect of the training might only be detectable in the combined parameter, while for $\mathrm{T}$ or $\mathrm{C}$ alone a larger sample size or longitudinal data would be required. Furthermore, we replicated prior results, which showed Tai chi qigong training to improve psychosocial factors in older individuals. ${ }^{18}$ No significant associations with TYr or TWk were detected with regard to sexual health. However, negative trends emerged for TWk and sexual dysfunctions indicating larger a sample size might have revealed significant associations. When interpreting these results, it is important to consider some limitations of the study. First, the small sample size limits a generalization to a larger population and a replication in a larger sample is required. Further, causal inferences are not allowed since the data is of cross-sectional nature. Longitudinal data confirming a decline of the CT-ratio and depressive symptoms with an increase in training sessions of self-defense Kung-fu and Tai chi qigong are needed. Finally, salivary steroids sampled at 8:00 a.m. represent a point measure, while diurnal profiles with more sampling time points or hair steroids might be more adequate to reflect an individuals' hormonal state.

We here provide first results for a potential underlying biological mechanism through which training of Tai chi qigong and self-defense Kung-Fu might cause its overall beneficial effects in aging men - namely by buffering age-related alterations in the HPA and HPG axes. 


\section{References}

1. Kirkwood TBL. Understanding the odd science of aging. Cell. 2005;120(4):437-447. doi:10.1016/j.cell.2005.01.027.

2. HAYFLICK L. Biological Aging Is No Longer an Unsolved Problem. Ann N Y Acad Sci. 2007;1100(1):1-13. doi:10.1196/annals.1395.001.

3. Baer N, Schuler D, Moreau-gruet F. Depressionen in Der Schweizer Bevölkerung Daten Zur Epidemiologie, Behandlung Und Sozial-Beruflichen Integration.; 2013.

4. Walther A, Ehlert U. Steroid secretion and psychological well-being in men 40+. In: $T$. Rice \& L. Sher (Eds.), Neurobiology of Men's Mental Health. New York, Nova. ; 2015:287-322.

5. Schiller CE, Johnson SL, Abate AC, et al. Reproductive Steroid Regulation of Mood and Behavior. Compr Physiol. 2016;6(July):1135-1160. doi:10.1002/cphy.c150014.

6. Feldman $\mathrm{H}$ a., Longcope C, Derby $\mathrm{C}$ a., et al. Age trends in the level of serum testosterone and other hormones in middle-aged men: Longitudinal results from the Massachusetts Male Aging Study. J Clin Endocrinol Metab. 2002;87(2):589-598. doi:10.1210/jc.87.2.589.

7. Walther A, Phillip M, Lozza N, Ehlert U. The rate of change in declining steroid hormones : a new parameter of healthy aging in men ? Oncotarget. 2016;7(38):1-28.

8. Karlamangla AS, Friedman EM, Seeman TE, Stawksi RS, Almeida DM. Daytime trajectories of cortisol : Demographic and socioeconomic differences — Findings from the National Study of Daily Experiences. Psychoneuroendocrinology. 2013. doi:10.1016/j.psyneuen.2013.06.010.

9. Nater UM, Hoppmann CA, Scott SB. ScienceDirect Diurnal profiles of salivary cortisol and alpha-amylase change across the adult lifespan : Evidence from repeated daily life assessments. Psychoneuroendocrinology. 2013;38(12):3167-3171. doi:10.1016/j.psyneuen.2013.09.008.

10. Johnson JM, Nachtigall LB, Stern TA. The Effect of Testosterone Levels on Mood in Men: A Review. Psychosomatics. 2013;54(6):509-514. doi:10.1016/j.psym.2013.06.018.

11. Walther A, Mahler F, Debelak R, Ehlert U. Psychobiological Protective Factors Modifying the Association Between Age and Sexual Health in Men : Findings From the Men's s Health $40+$ Study. 2017. doi:10.1177/1557988316689238.

12. Snyder PJ, Bhasin S, Cunningham GR, et al. Effects of Testosterone Treatment in Older Men. N Engl J Med. 2016;374(7):611-624. doi:10.1056/NEJMoa1506119. 
13. Walther A, Rice T, Kufert Y, Ehlert U. Neuroendocrinology of a Male-Specific Pattern for Depression Linked to Alcohol Use Disorder and Suicidal Behavior. Front Psychiatry. 2017;7(January):1-9. doi:10.3389/fpsyt.2016.00206.

14. Sollberger S, Ehlert U. How to use and interpret hormone ratios. Psychoneuroendocrinology. 2015. doi:10.1016/j.psyneuen.2015.09.031.

15. Ehlert U, Gaab J, Heinrichs M. Psychoneuroendocrinological contributions to the etiology of depression, posttraumatic stress disorder, and stress-related bodily disorders: The role of the hypothalamus-pituitary-adrenal axis. Biol Psychol. 2001;57(1-3):141-152. doi:10.1016/S0301-0511(01)00092-8.

16. Irwin MR, Olmstead R, Motivala SJ. Improving Sleep Quality in Older Adults with Moderate Sleep Complaints : A Randomized Controlled Trial of Tai Chi Chih. 2005.

17. Nyman SR, Skelton DA. The case for Tai Chi in the repertoire of strategies to prevent falls among older people. Perspect Public Health. 2016.

18. Chan AWK, Yu DSF. Effects of tai chi qigong on psychosocial well-being among hidden elderly, using elderly neighborhood volunteer approach : a pilot randomized controlled trial. 2017:85-96.

19. Ho RTH, Fong TCT, Wan AHY, et al. A randomized controlled trial on the psychophysiological effects of physical exercise and Tai-chi in patients with chronic schizophrenia. 2016;171:42-49. doi:10.1016/j.schres.2016.01.038.

20. Campo RA, Light KC, Connor KO, et al. Blood pressure , salivary cortisol , and inflammatory cytokine outcomes in senior female cancer survivors enrolled in a tai chi chih randomized controlled trial. 2015:115-125. doi:10.1007/s11764-014-0395-x.

21. Jung S, Lee E, Lee S, Kim M, Lee MS. Tai Chi for Lower Urinary Tract Symptoms and Quality of Life in Elderly Patients with Benign Prostate Hypertrophy: A Randomized Controlled Trial. 2012;2012. doi:10.1155/2012/624692.

22. Radloff L. The CES-D scale a self-report depression scale for research in the general population. Appl Psychol Meas. 1977. doi:10.1177/014662167700100306.

23. Hautzinger M, Bailer M, Hofmeister D, Keller F. Allgemeine Depressionsskala (ADS). Psychiatr Prax. 2012;39(6):302-304.

24. Glaesmer H, Grande G, Braehler E, Roth M. The German version of the satisfactionwith life scale (SWLS) psychometric properties, validity, and populationbased norms. Eur J Psychol Assess. 2011;27(2):127-132. doi:10.1027/1015$5759 / \mathrm{a} 000058$.

25. Rosen, Wagner, Riley. A multidimensional scale for assessment of erectile 
dysfunction. Urology. 1997;49(97):822-830.

26. Wiltink J, Hauck EW, Phädayanon M, Weidner W, Beutel ME. Validation of the German version of the International Index of Erectile Function (IIEF) in patients with erectile dysfunction, Peyronie's disease and controls. Int J Impot Res. 2003;15(3):192197. doi:10.1038/sj.ijir.3900997.

27. Chen C, Nakagawa S, An Y, Ito K, Kitaichi Y, Kusumi I. The exercise-glucocorticoid paradox: How exercise is beneficial to cognition, mood, and the brain while increasing glucocorticoid levels. Front Neuroendocrinol. 2017;44:83-102. doi:10.1016/j.yfrne.2016.12.001.

28. Sato K, Iemitsu M, Katayama K, Ishida K, Kanao Y, Saito M. Responses of sex steroid hormones to different intensities of exercise in endurance athletes. Exp Physiol. 2016;101(1):168-175. doi:10.1113/EP085361. 


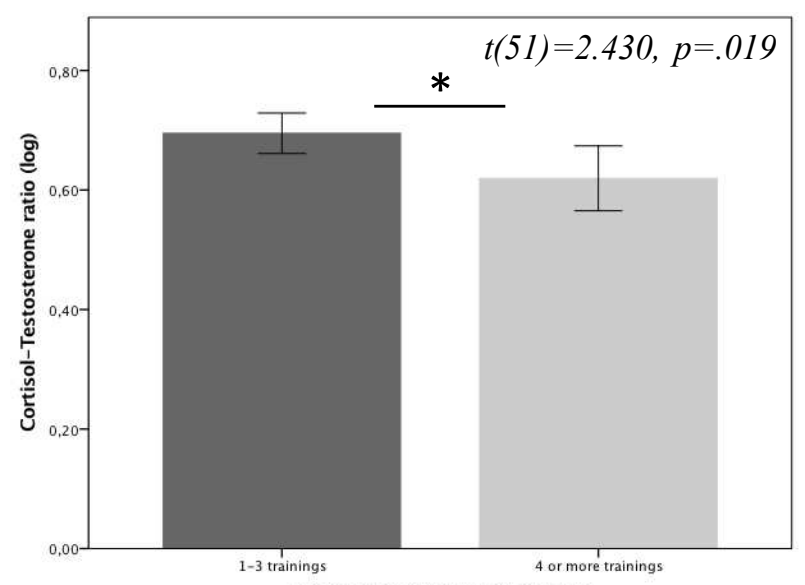

Tai chi qigong / Kung-fu training

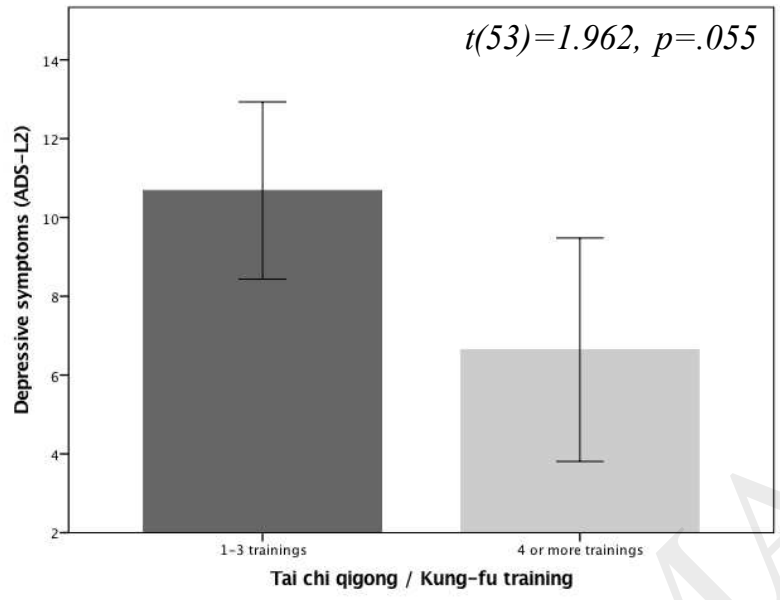

Fig. 1. T-tests for the groups 1-3 trainings per week versus 4 or more trainings per week for the CT-ratio (top) and depressive symptoms (bottom). 


\begin{tabular}{|c|c|c|c|c|}
\hline \multicolumn{3}{|c|}{ Table 1. Sample characteristics } & & \\
\hline \multicolumn{2}{|l|}{ Characteristic } & $\mathrm{n}$ & $\mathrm{M}(\mathrm{SD})$ & $\%$ \\
\hline \multicolumn{2}{|l|}{ Age } & 111 & $49.8(7.9)$ & \\
\hline \multicolumn{2}{|l|}{$\operatorname{BMI}\left(\mathrm{kg} / \mathrm{m}^{2}\right)$} & 103 & $25.5(3.4)$ & \\
\hline \multicolumn{2}{|l|}{ Cortisol (nmol/L) } & 98 & $16.6(6.8)$ & \\
\hline \multicolumn{2}{|l|}{ Testosterone (nmol/L) } & 103 & $74.2(28.7)$ & \\
\hline \multicolumn{2}{|c|}{ Cortisol-Testosterone-Ratio (log) } & 98 & $-1.5(0.5)$ & \\
\hline \multicolumn{2}{|c|}{ Medication intake (No/Yes) } & $93 / 18$ & & $83.8 / 16.2$ \\
\hline $\begin{array}{l}\text { Smoking status } \\
\text { (No/Yes) }\end{array}$ & $87 / 24$ & & $78.4 / 21.6$ & \\
\hline Training per week (in h) & 56 & $4.4(4.9)$ & & \\
\hline Years of training & 56 & $10.8(8.7)$ & & \\
\hline \multicolumn{4}{|l|}{ Training type } & \\
\hline Tai chi qigong & 18 & & 32.1 & \\
\hline $\begin{array}{l}\text { Self-defense Kung-Fu } \\
\text { (Wing-chun, Eskrima) }\end{array}$ & 19 & & 33.9 & \\
\hline Both training types & 19 & & 33.9 & \\
\hline & & & $\Delta$ & \\
\hline
\end{tabular}

